\title{
EDITORIAL
}

\section{Idiopathic pulmonary fibrosis: a treatable disease?}

\author{
U. Costabel
}

Idiopathic pulmonary fibrosis (IPF) is a chronic progressive lung disease characterised by fibroblast proliferation and extracellular matrix remodelling. The usual treatment strategy is anti-inflammatory, but this is unsuccessful in preventing the fatal outcome in most patients. The disorder is largely unresponsive to corticosteroids and immunosuppressants. Several papers have recently highlighted the prognostic importance of the new histological classification of the idiopathic interstitial pneumonia, which limits the definition of IPF to the histopathological pattern of usual interstitial pneumonia (UIP) and discriminates several other subgroups with a better prognosis [1-3]. IPF is the most common and most aggressive variant of the chronic idiopathic interstitial pneumonias. One of the hallmarks of IPF/UIP is the formation of multiple patchy fibroblastic foci that evolve to fibrosis, in contrast with all the other entities of the idiopathic interstitial pneumonia.

For decades, the leading hypothesis with regards to the pathogenesis of IPF was that the initial event is an inflammatory process (alveolitis characterised by infiltration of inflammatory cells such as macrophages, lymphocytes and neutrophils). Inflammation would lead to recruitment of fibroblast/myofibroblasts and finally to collagen formation and irreversible fibrosis. The new concept is that IPF is a primary epithelial/ fibroblastic disease, and inflammation is nowadays considered to be the secondary and not the primary event [4]. This new hypothesis is the basis for understanding the disappointing results with an isolated anti-inflammatory treatment. New strategies are targeted at developing truly antifibrotic drugs.

Interferon (IFN)- $\gamma$ is one of several antifibrotic drugs considered for investigation in IPF. This is based on the concept that IPF seems to be characterised by a predominant expression of T-helper cell (Th) type- 2 cytokines, such as interleukin (IL)-4 and IL-5 [5, 6]. IFN- $\gamma$ may shift the Th1/ Th2 balance towards a Th1 profile. This cytokine also inhibits fibroblast proliferation and collagen synthesis and antagonises the effects of transforming growth factor- $\beta$ [7, 8]. A recent study by ZIESCHE et al. [9] investigated this concept in a small phase-II trial in IPF. The results raised initial hope but also considerable criticism and discussion concerning the efficacy of IFN- $\gamma$. These authors performed a randomised trial of 18 highly selected patients with IPF who had no responses to previous immunosuppressive therapy. Nine patients treated for 12 months with IFN- $\gamma_{1 b}$ plus low-dose prednisolone showed an improvement in lung function, whereas the nine patients in the group given prednisolone alone deteriorated in lung function. It is remarkable to see such clearly defined differences in the change in lung function in an IPF trial, which may be entirely due to the small cohort of patients. It was also remarkable that all patients finished the 12-month trial. None of them had to be withdrawn for side-effects and none of them died. A reanalysis of this study on a case-by-case basis showed that not all patients had IPF

Correspondence: U. Costabel, Ruhrlandklinik, Dept of Pneumology/ Allergy, Tueschener Weg 40, D-45239 Essen, Germany. Fax: 49 2014334029. E-mail: erj.costabel@t-online.de according to the new definition (G. Raghu, Division of Pulmonary and Critical Care Medicine, University of Washington Medical Center, Seattle, WA, USA; personal communication). Nevertheless, this study raised hope and a demand for IFN- $\gamma$ treatment in patients with IPF requesting the off-label treatment with this drug, despite considerable costs and still unproven efficacy.

In this issue of the European Respiratory Journal, PRASSE et al. [10] report their own experience with five patients with IPF, treated in a similar way to the patients in the study by ZIESCHE et al. [9], with IFN- $\gamma$ and low-dose prednisolone. They could not confirm the previous optimistic results. Only one of their patients showed an improvement in pulmonary function, while four patients deteriorated. In addition, two patients declined further therapy after 4 months because of side-effects, mainly severe flu-like symptoms, following the injection of IFN- $\gamma_{1 \mathrm{~b}}$. Admittedly, the paper published in this issue of the $E R J$ is based on an even smaller series of cases than the study by ZIESCHE et al. [9], such that it has to be considered as an anecdotal report. Nevertheless, the editors of the $E R J$ felt that the observation is worth publishing, since the data are contradicting that shown by ZIESCHE et al. [9].

Is there any explanation for the different results? One important difference may be the severity of disease, as expressed in the degree of impairment of lung function. In this regard, there was a significant difference in the mean value of the total lung capacity before study, which was $57 \pm 9 \%$ predicted in the present paper, but $70 \pm 10 \%$ in the paper by ZIESCHE et al. [9]. This may indicate that IFN- $\gamma$ only acts in early disease, and that patients with far-advanced disease, particularly those with end-stage fibrosis, take no benefit from the drug. Along these lines, it is of interest that early this year, French authors described four patients with IPF from a cohort of 10 patients who developed acute respiratory distress syndrome in an explosive manner, and eventually died from this complication, in close temporal relation to IFN- $\gamma$ therapy [11]. All these patients had endstage pulmonary fibrosis (total lung capacity of $<45 \%$ pred or carbon monoxide diffusion capacity of $<30 \%$ pred).

At this point of the discussion, it is worth looking at the data from the recently completed phase-III clinical trial of IFN- $\gamma_{1 b}$ injection. The preliminary data have been presented at the recent European Respiratory Society and American Thoracic Society meetings. A total of 330 patients were randomised to receive either placebo or IFN- $\gamma_{1 \mathrm{~b}}$ for a minimum of $1 \mathrm{yr}$. The drug was well tolerated, with only few discontinuations due to adverse events. The primary endpoint was progression-free survival, and this was not significantly different between the treated group and the placebo group. An explorative analysis showed a nonsignificant reduction in mortality in favour of IFN- $\gamma$ for the whole study population, but a significant decrease in mortality in those patients with a forced vital capacity of $>55 \%$ at study entry. These data indicate, that IFN- $\gamma$ may be beneficial only in patients with mild-to-moderate disease. The definitive proof of efficacy will come from a further, even larger, trial 
with IFN- $\gamma$ given for $\geqslant 2$ yrs to such a subgroup of IPF patients, with survival time as the primary end-point. This trial is likely to start soon.

For the moment, the off-label use of this drug and other antifibrotic substances that have not been approved cannot be recommended. Potential opportunities for therapeutic interventions in the future include, besides interferon- $\gamma$, the use of other antifibrotic agents such as pirfenidone, endothelinreceptor antagonists, and antagonists to growth factors or to cytokines, such as tumour necrosis factor- $\alpha$. The antioxidant $N$-acetylcysteine has currently been tested in a multinational clinical trial, and the results can be expected in early 2004. The treatment of idiopathic pulmonary fibrosis in the future may be similar to the treatment of cancer. It is conceivable that a multidrug regimen may be used, composed of an antifibrotic drug to attack the fibroblastic foci, plus immunosuppressants to inhibit the secondary inflammation, plus an antioxidant to protect against the oxidative mechanisms. Using this approach, idiopathic pulmonary fibrosis may become a treatable disease.

\section{References}

1. Bjoraker JA, Ryu JH, Edwin MK, et al. Prognostic significance of histopathologic subsets in idiopathic pulmonary fibrosis. Am J Respir Crit Care Med 1998; 157: 199-203.

2. Flaherty KR, Toews GB, Travis WD, et al. Clinical significance of histological classification of idiopathic interstitial pneumonie. Eur Respir J 2002; 19: 275-283.

3. Travis WD, King TE, Bateman ED, et al. ATS/ERS international multidisciplinary consensus classification of idiopathic interstitial pneumonias. General principles and recommendations. Am J Respir Crit Care Med 2002; 165: 277-304.

4. Selman M, King TE Jr, Pardo A. Idiopathic pulmonary fibrosis: prevailing and evolving hypotheses about its pathogenesis and implication for therapy. Ann Intern Med 2001; 134: 136-151.

5. Majumdar S, Li D, Ansari T, et al. Tissue cytokine profiles of cryptogenic fibrosing alveolitis (CFA) and fibrosing alveolitis associated with systemic sclerosis (FASSc) are distinct: a quantitative in situ study of open lung biopsies. Eur Respir J 1999; 14: 251-257.

6. Prior C, Haslam PL. In vivo levels and in vitro production of interferon-gamma in fibrosing interstitial lung diseases. Clin Exp Immunol 1992; 88: 280-287.

7. Gurujeyalakshmi G, Giri SN. Molecular mechansims of antifibrotic effect of interferon gamma in bleomycin-mouse model of lung fibrosis: down-regulation of TGF-beta and procollagen I and III gene expression. Exp Lung Res 1995; 21: 791-808

8. Eickelberg O, Pansky A, Koehler E, et al. Molecular mechanisms of TGF-(beta) antagonism by interferon (gamma) and cyclosporine A in lung fibroblastis. FASEB $J$ 2001; 15: 797-806.

9. Ziesche R, Hofbauer E, Wittmann K, Petkov V, Block LH. A preliminary study of long-term treatment with interferon gamma-1b and low-dose prednisolone in patients with idiopathic pulmonary fibrosis. $N$ Engl J Med 1999; 341: 1264-1269.

10. Prasse A, Müller K-M, Kurz C, Hamm H, Virchow JC Jr. Does interferon- $\gamma$ improve pulmonary function in idiopathic pulmonary fibrosis? Eur Respir J 2003; 22: 906-911.

11. Honoré I, Nunes $\mathrm{H}$, Groussard $\mathrm{O}$, et al. Acute respiratory failure after interferon- $\gamma$ therapy of end-stage pulmonary fibrosis. Am J Respir Crit Care Med 2003; 167: 953-957. 